\title{
Der Schutz des Beschuldigten vor Selbstbelastung in den USA - ein europäisches Vorbild?
}

Andreas Bonnen

Im Zuge der Vereinheitlichung des europäischen Strafprozessrechts stellt sich mehr und mehr die Frage, ob eine Symbiose aller europäischen Strafprozesssysteme und den damit verbundenen vielschichtigen Veränderungen in den Ländern selbst möglich wäre, oder eine (Teil-) Übernahme des Strafprozessrechts der USA, dass in seiner Ausgestaltung bereits weitreichend und umfassend ist, und auch im Rahmen einer globalen Zusammenarbeit mit den USA Vorteile bringen könnte, herangezogen werden sollte. Dieses System soll am Beispiel des Schutzes vor Selbstbelastung kurz beleuchtet werden.

\section{Ursprung und geschichtliche Entwick- lung des Schutzes vor Selbstbelastung}

m nordamerikanischen Raum existierte bis ins späte 16. Jahrhundert der aus England stammende und durch kirchliche Gerichte geführte Inquisitionsprozess. 1 Der Angeklagte hatte die Pflicht, notfalls unter Eid gegen sich selbst aussagen zu müssen und sich selbst eines Verbrechens zu bezichtigen. Die Aussage wurde auch mit Gewalt erzwungen. Im 17. Jahrhundert bildete sich mehr und mehr eine breite Front gegen diese Art der Prozessführung und gegen das englische Regime. Es verbreitete sich eine zunächst ungeschriebene Maxime, die von jedem Gericht beachtet werden sollte: nemo tenetur se ipsum prodere (accusare). 2 Niemand sollte dazu gezwungen werden, sich selbst vor einem Gericht zu belasten oder unter Eid gegen sich selbst aussagen zu müssen. Zugleich nahmen aber die Rechtsentwicklungen im nordamerikanischen Raum und in Europa, speziell in England, unterschiedliche Entwicklungen und lösten sich voneinander.

\section{1. nemo tenetur Grundsatz: keine bela- stende Aussage gegen sich selbst}

Dieses amerikanische nemo- tenetur- Prinzip hatte zunächst nur den Zweck sicherzustellen, dass ein Beschuldigter keine Angaben zu den gegen ihn erhobenen Vorwürfen machen brauchte, bevor nicht eine Anklage (indictment) einer grand jury vorlag. ${ }^{3}$ Im Laufe der Zeit wurde dieser Grundsatz durch die Rechtsprechung und durch die verschiedenen, im Wege der Unabhängigkeitsbestrebungen geschaffenen einzelstaatlichen Verfassungen, immer weiter ausgebaut und schließlich durch das Drängen verschiedener Einzelstaaten im Jahre 1789 bundesstaatlich im Bill of Rights im Amendment $V^{4}$ gesetzlich verankert. ${ }^{5}$ Über die sogenannte »due process of law- Klausel ${ }^{6}$ (rechtliches Gehör) des Amendment XIV7 , die sich aus- drücklich an alle Einzelstaaten richtet $^{8}$, wurde das Privileg des Schutzes vor Selbstbelastung, wie auch viele andere Rechte des Bill of Rights, durch die Rechtsprechung auf alle einzelstaatlichen Strafverfahren für anwendbar erklärt. Dieser Weg über den Supreme Court war jedoch schwierig. Im Gegensatz zu Bundesgerichten hat er gegenüber Gerichten der Einzelstaaten weniger Rechte und kann Schuldsprüche nur aufheben, wenn er einen Verstoß gegen die due process of law Klausel erkennt. ${ }^{9}$ Als Folge dieser Entwicklung ist der nemo- tenetur Grundsatz heute in jedem Anklageverfahren zu beachten. Dies bedeutet jedoch nicht, dass durch die Aufnahme des nemo- tenetur- Grundsatzes der Strafprozess unmittelbar vollständig geändert wurde. Vielmehr bedurfte es einer langen Entwicklung den gedachten Beschuldigtenschutz auch verwirklichen zu können.

Das amerikanische Strafverfahrensrecht steht durch die Supreme Court Rechtsprechung in besonderer Weise unter dem Einfluss der Bill of Rights in den ersten zehn Zusatzartikeln und der Rechte aus dem 14. Zusatzartikel. Geprägt wurde das Strafverfahren neben zahlreichenden Urteilen des Supreme Court aber insbesondere durch den liberalen Chief Justice Earl Warren, der in den Jahren 1953 bis 1969 dieses leitende Amt inne hatte. Der Supreme Court legte während dieser Amtszeit die durch die Verfassung gegebenen Garantien weit aus und bildete durch seine zahlreichen Entscheidungen ein durch Auslegung geformtes Strafprozessrecht. ${ }^{10}$ In der Zeit nach Warren wechselte im Rahmen von Neuernennungen der Richter auch die liberale $\mathrm{Zu}$ sammensetzung des Supreme Court. Die umwälzenden Reformen auf dem Gebiet des Beschuldigtenschutzes und des Strafprozesses wurden nicht fortgeführt. Unter dem Einfluss der konservativen Zusammensetzung und unter der Leitung des Chief Justice Warren Earl Burger (1969 bis 1986) und später unter William $\mathrm{H}$. Rehnquist wurden die liberalen Ansätze beschränkt. ${ }^{11}$

\section{Voluntariness Test: Freiwilligkeit der Aussage}

Seit dem Jahre 1936 galt im amerikanischen Strafverfahren neben dem nemo- teneturGrundsatz der sogenannte voluntariness test, der ebenfalls über die due process- Klausel des Amendment XIV auch für die Einzelstaaten verbindlich vorgeschrieben war. ${ }^{12}$ Nach diesem voluntariness test sollte ein Geständnis trotz einer möglichen Selbstbelastung durch den Beschuldigten verwertbar sein, solange es freiwillig abgegeben worden war. Der Test bezog sich auf die Glaubhaftigkeit des abgelegten Geständnisses. ${ }^{13}$ Waren die angewendeten Vernehmungsmethoden der Polizei, der Staatsanwaltschaft oder des Richters dazu geeignet, dem Beschuldigten ein falsches Geständnis zu entlocken, sollte dieses Geständnis im folgenden Prozess nicht verwertbar sein. In der Folgezeit wurde in verschiedenen leading decisions (Präzedenzfällen) der Schutz vor Selbstbelastung entgegen des voluntariness tests weiter ausgebaut. In der Entscheidung Brown vs. Mississippi aus dem Jahre 1936 wurden mehrere farbige Beschuldigte wegen Mordes zum Tode verurteilt wurden. ${ }^{14}$ Die Geständnisse waren durch schlimmste Folterungen seitens der Polizei erzwungen worden. Der Supreme Court hob die Urteile auf und stützte sich dabei auf das Amendment XIV wonach durch die voluntariness test- Rechtsprechung ein Fall eines erzwungenen Geständnisses vorlag. In der Folgezeit wurden Geständnisse grundsätzlich als unzulässig angesehen, die unter Drohungen, Schlafentzug oder Dauerverhören entstanden. ${ }^{15} \mathrm{Zu}$ berücksichtigen waren auch immer das Alter des Beschuldigten, seine geistige Entwicklung und seine Bildung. ${ }^{16}$ Bei Vorliegen einer oder mehrerer dieser Merkmale konnte das Geständnis vom Gericht als unfreiwillig angesehen und durfte nicht verwertet werden. Der voluntariness test entwickelte sich aber durch diese zahlreichen Prüfungsmerkmale immer mehr in einen allgemeinen fairness test. ${ }^{17}$ Es entstand keine allgemein verbindliche Regelung, wie ein Beschuldig- 
ter zu vernehmen und zu behandeln war. Sowohl die vernehmenden Beamten als auch das angerufene Gericht hatten jeweils erneut über die $\mathrm{Zu}$ lässigkeit der angewendeten Vernehmung $\mathrm{zu}$ entscheiden und die Verwertbarkeit zu prüfen.

\section{Escobedo vs. Illinois: Recht auf Verteidigerbeistand}

Im Laufe der Entwicklung der Rechtssprechung erging auch die bahnbrechende Entscheidung im Fall Escobedo vs. Illinois aus dem Jahre 1964. ${ }^{18}$ Der Beschuldigte Danny Escobedo war wegen Mordverdachts festgenommen und verhört worden. $\mathrm{Zu}$ Beginn dieses Verhörs verlangte er einen Anwalt zu sprechen, was man ihm trotz der Anwesenheit seines Verteidigers auf dem Polizeirevier verweigerte. Nach einem mehrstündigen Verhör, bei dem Escobedo fortwährend stehen musste, wurde ihm mitgeteilt, er dürfe nach Hause gehen, wenn er seinen Mittäter verrate und dieser so verurteilt werden könne. Diese eigentlich für ihn günstige Sachlage verweigerte Escobedo vor dem Hintergrund, dass sein Anwalt ihm Tage vor der Verhaftung mitgeteilt habe, er solle nie eine Aussage machen, ohne vorher mit ihm gesprochen zu haben. Escobedo gestand zuletzt selbst die Tat. Der Supreme Court hielt das Geständnis für unverwertbar und stützte sich dabei auf das Amendment VI. ${ }^{19}$ Sein Recht auf Verteidigerbeistand sei verletzt worden und aus diesem Grunde sei die Aussage nicht freiwillig gewesen. Auch war die Aussage Escobedos durch die für ihn neue Haftsituation ohne die Hilfe eines Rechtsbeistandes unter Druck und damit unfreiwillig zustande gekommen. Es lag somit auch ein Verstoß gegen den nemo- tenetur Grundsatz vor. Der Supreme Court führte dann allgemein weiter aus, dass in vielen Fällen ein Geständnis mit List erzwungen werde und so unverwertbar sein könnte.

Das in dieser Entscheidung gefestigte Recht der Verteidigerkonsultation hängt folglich eng mit dem Schutz vor Selbstbelastung durch dem nemo tenetur Grundsatz zusammen und ergänzt den allgemeinen Beschuldigtenschutz weiter. ${ }^{20}$ Bei einer Vernehmung musste in Folge dieser Entscheidung nun einem Beschuldigten der Kontakt mit seinem Verteidiger gestattet werden. Die Beamten hatten ihn auf eine Konsultationsmöglichkeit hinzuweisen. Eine genaue Ausgestaltung des Rechts wurde vom Gericht jedoch nicht vorgenommen.

\section{Miranda vs. Arizona21: Festlegung der Beschuldigtenrechte}

Schon 2 Jahre später, im Jahre 1966, erging eine weitere Entscheidung im Bezug auf den Schutz des Beschuldigten vor Selbstbelastung. In der Entscheidung Miranda vs. Arizona wurde der Beschuldigte Ernesto Miranda wegen Raubes und Kidnapping verurteilt. Sein Geständnis legte er nach einem mehrstündigen Verhör auf einem Polizeire- vier ab. Er wurde dabei zu keinem Zeitpunkt von einem Beamten über seine Rechte belehrt. Der Beschuldigte unterschrieb aber am Ende des Verhörs ein Geständnis, in dem er versicherte, über seine Rechte vollständig in Kenntnis gesetzt worden zu sein, und dass er das Geständnis freiwillig abgegeben habe. Der Supreme Court hob die Entscheidung und die mit diesem Fall verbundenen ähnlich gelagerten Fälle Vignera vs. New York ${ }^{22}$, Westover vs. United States ${ }^{23}$ und California vs. Stewart ${ }^{24}$, in denen ebenfalls ein Geständnis ohne einen Hinweis über die Beschuldigtenrechte durch verschiedene Tricks erzwungen wurde, auf. Das Gericht stellte fest, dass Miranda vor seinem Geständnis nicht ordnungsgemäß über seine ihm zustehenden verfassungsmäßigen Rechte informiert worden sei. Zudem sei die Vernehmungssituation derart belastend gewesen, dass sein Recht, sich nicht selbst belasten zu müssen, verletzt wurde. Gestützt wurde diese Entscheidung erstmalig auf das Amendment $\mathrm{V}^{25}$ und zusätzlich auf die due process- Klausel des Amendment XVI.

Der Supreme Court beschrieb im Urteil jedoch auch erstmalig, wie eine Belehrung aus Sicht des Gerichts zu erfolgen habe, um die darauf folgende Aussage des Beschuldigten in einem Prozess vollständig verwerten zu können. Es entstanden die sogenannten »Miranda Warnings « 26 , die heute einem Beschuldigten vor jedem ersten Verhör mitzuteilen sind. In der Praxis hat sich die Verlesung der Rechte unmittelbar bei der Festnahme etabliert, damit im Falle einer spontanen Aussage bei der

Festnahme die Aussage in einem Prozess verwertbar ist. Frei übersetzt lauten die Rechte im wie folgt 27 .

- das Recht, die Aussage über die gegen ihn erhobenen Vorwürfe zu verweigern,

- das Recht, sich während oder vor der Vernehmung von einem Anwalt beraten zu lassen,

- die Übernahme der Bezahlung des Anwalts auf Staatskosten bei Mittellosigkeit,

- dass jede Aussage des Beschuldigten in einem Prozess gegen ihn verwendet werden kann.

Diese Miranda- Warnings wurden durch das Urteil zwingende Voraussetzung für die Verwertung eines Geständnisses in einem Gerichtprozess. In der Entscheidung wird aber auch deutlich, dass eine derartige Belehrung nur erfolgen muss, wenn der Beschuldigte verhaftet wurde oder in sonstiger Weise einer freiheitsentziehenden Maßnahme ausgesetzt ist. ${ }^{28}$ Liegt eine andere Art der "Vernehmung « vor, bleibt weiterhin der immer noch geltende voluntariness test anwendbar. ${ }^{29}$ Ein Geständnis ist dann auch ohne eine Belehrung über die Rechte verwertbar, solange es freiwillig abgelegt wurde.

\section{Entwicklung nach der Miranda- Entscheidung}

Im Zuge der Rechtsprechung unter Chief Justice Warren wurden diese herausgehobenen Grund- sätze der Miranda- Entscheidung zunächst ausgebaut und verfeinert. ${ }^{30}$ dies bedeutete dies jedoch nicht, dass die eigentlichen Beschuldigtenrechte weiter gefestigt wurden. Chief Justice Warren wurde 1969 nicht wieder ernannt und durch Chief Justice Warren E. Burger ersetzt. Während dieser Amtsperiode fand keine Abkehr von den Miranda- Rechten statt, aber der eingeleitete Umbruch der Rechtsprechung zugunsten der Beschuldigtenrechte stagnierte. Miranda wurde wieder »abgemildert «. ${ }^{31}$ Dieser Trend zugunsten erleichterter Strafverfolgung wurde auch in den Folgejahren fortgesetzt. ${ }^{32}$

Einen einschneidenden Eingriff in diese Beschuldigtenrechte stellte die Verabschiedung des 18 U.S.C. $§ 3501$ im Jahre 1968 dar. ${ }^{33}$ Dieser beinhaltete unter Berufung auf den bisher geltenden voluntariness test, dass Geständnisse in jedem Verfahrensstadium verwertbar sind, solange sie freiwillig abgegeben werden. Zudem wurden Fallbeispiele aufgezählt, unter deren Voraussetzungen Geständnisse als nicht freiwillig anzusehen sind. Eine Belehrung im Sinne der Miranda- Warnings war nicht mehr zwingend erforderlich, solange die Ermittlungsbeamten belegen konnten, dass das Geständnis freiwillig im Sinn des $§ 3501$ abgegeben wurde. Sinn und Zweck dieses Gesetzes war es, eine Harmonisierung der Vorschriften über den nemo- tenetur Grundsatz, den voluntariness test und die Rechtsprechung des Supreme Court zu erreichen und den Ermittlungsbehörden eine Strafverfolgung ohne strenge Formvorschriften zu ermöglichen.

Eine völlige Abkehr von der Miranda- Rechtsprechung stellte dann zwangsläufig auch eine Entscheidung des 4th Circuit Court of Appeals dar. Der Beschuldigte wurde weder belehrt, noch kannte er seine Rechte, legte aber trotzdem ein Geständnis ab. Nach Auffassung des Gerichts war dieses Geständnis freiwillig im Sinne des § 3501 und nicht erzwungen. Der Beschuldigte berief sich hingegen auf seine Beschuldigtenrechte aus der Miranda- Entscheidung und den nemotenetur- Grundsatz. Dieser Fall Dickerson vs. United States ${ }^{34}$ ging durch die Instanzen bis vor den Supreme Court, der nun zu entscheiden hatte, ob der 18 U.S.C. § 3501 verfassungsgemäß ist.

\section{Dickerson vs. United States: Bestätigung Miranda- Warnings}

Der Angeklagte Charles Thomas Dickerson war wegen bewaffneten Raubüberfalls festgenommen und durch das FBI verhört worden. Bei dieser Vernehmung legte er ein umfassendes Geständnis $\mathrm{ab}$, allerdings ohne vorher durch das FBI über seine Rechte belehrt worden zu sein. Es lag demnach eindeutig ein Verstoß gegen die MirandaWarnings vor. Auch war das Geständnis nicht als freiwillig im Sinne des voluntariness test anzusehen, da Dickerson ein Geständnis im Rahmen einer offiziellen Vernehmung ablegte. Dickerson 
wurde jedoch schuldig gesprochen und zu einer langen Gefängnisstrafe verurteilt. Das Gericht berief sich auf die Freiwilligkeit des Geständnisses im Sinne des $\S 3501$. In der ersten Rechtsmittelinstanz vor dem 4th Circuit Court of Appeals bestätigten die Richter die Verurteilung Dickersons. Letztinstanzlich hob der Supreme Court im Jahre 2000 die Verurteilung von Dickerson auf und stützte sich dabei auf die in den Amendments $\mathrm{V}$ und XIV und in der Miranda- Entscheidung festgelegten Beschuldigtenrechte. Zugleich wurde für den Staat und seine Einzelstaaten festgelegt, dass die Miranda- Warnings ein verfassungsmäßiges Recht ${ }^{35}$ seien und nicht durch Gesetze, wie hier der $\S 3501$, umgangen oder unterlaufen werden können. ${ }^{36}$ Der $\S 3501$ ist nach dieser Entscheidung rechtswidrig und nicht mit der Verfassung zu vereinbaren. Die sehr knappe 7 zu 2 Entscheidung lässt allerdings in der Begründung weitere Fragen offen. Zum einen ist der Text der MirandaWarnings als nicht bindend bezeichnet worden und zum anderen bleibt es weiterhin beim sogenannten voluntariness test, wenn keine Vernehmung im eigentlichen Sinn vorliegt. ${ }^{37}$ Es ist nicht deutlich geworden, warum sich die Richter für die Einstufung der Miranda- Warnings als Verfassungsrecht entschieden haben. Für die Zukunft besteht weiterhin eine gewisse Unsicherheit, welche legalen Tricks möglich sind, einen Beschuldigten zu überführen.

\section{Grenzen des Schutzes vor Selbstbelastung}

Wurde der Beschuldigte ohne Belehrung vernommen und hat er während dieser Vernehmung die im zur Last gelegte Tat gestanden, kann das Geständnis nur verwertet werden, wenn eine erneute Vernehmung mit vorangehender Belehrung erfolgt und der Beschuldigte dort sein Geständnis bestätigt. Allerdings ist er dann während der Belehrung darauf hinzuweisen, dass das erste Geständnis nicht verwertet werden kann und es ihm frei stehe, das Geständnis zu bestätigen (qualifizierte Belehrung). ${ }^{38}$ Aus seinem Schweigen nach der Belehrung können dann keine Rückschlüsse für das Verfahren gezogen werden. ${ }^{39}$ Verzichtet der Beschuldigte hingegen nach einer ordnungsgemäßen Belehrung auf seine ihm zustehenden Rechte und sagt er aus, kann das Geständnis in der Hauptverhandlung gegen ihn verwertet werden. ${ }^{40}$ Eine Überprüfung dieses Verzichts findet in der Form statt, ob der Verzicht freiwillig zustande gekommen ist und der Beschuldigte die Konsequenzen des Verzichts verstanden hat. ${ }^{41}$ Des Weiteren kann in Fällen ein Geständnis ohne Belehrung verwertbar sein, wenn ein harmloser Verstoß vorliegt und dieser den Schuldspruch nicht beeinflusst hat (harmless- error- rule). ${ }^{42}$

Bereits vor der Miranda- Entscheidung hatte das Oberste Gericht in verschiedenen Entscheidungen ${ }^{43}$ festgehalten, dass sich der Schutz vor Selbstbelastung nur auf aktives Tun des Beschul- digten beziehe und nicht auch auf ein passives Dulden von Untersuchungsmaßnahmen. In der Praxis bedeutet dies, dass der Beschuldigte $\mathrm{zu}$ Photoaufnahmen und der Abnahme von Fingerabdrücken, Blut- und anderer Körperproben ${ }^{44}$ sowie zum Anziehen tatrelevanter Kleidung verpflichtet ist. ${ }^{45}$ Er kann diese eng begrenzten Formen der Ermittlung nicht unter dem Deckmantel des Schutzes vor Selbstbelastung verhindern. Unter bestimmten Umständen ist sogar bei Stimmoder Schriftproben eine aktive Mitwirkung des Beschuldigten möglich. Allerdings muss diese Untersuchung dem Zwecke der Identifizierung psychischer Eigenschaften des Beschuldigten dienen und nicht der Feststellung inhaltlicher Aussagen. Ein Sonderfall der Verwertung von Beweismitteln ohne vorherige Belehrung liegt in den Fällen bestimmter Gefahrsituationen für Polizeiund Ermittlungsbeamte vor. Ein Verdächtiger darf auch durchsucht oder befragt werden (search and seizure), wenn für die ermittelnden Beamten eine Notsituation vorliegt, zum Beispiel, um die Waffe des Verdächtigen sicherzustellen. Hier überwiegt das Schutzinteresse dem Schutz des Beschuldigten vor Selbstbelastung.

\section{Fruits of the poisonous tree doctrine und Verwertungsverbot}

Auf einen Verstoß gegen das nemo- tenetur- Prinzip oder gegen die Miranda- Warnings folgt im trial (Verfahren) immer ein exclusionary rule (Verwertungsverbot) für die durch den Verstoß erlangte Aussage. ${ }^{46}$ Ein solcher Verstoß hat aber auch weitreichende Konsequenzen für das weitere Ermittlungs- und Strafverfahren. Im Zuge der Entscheidungen New York vs. Quarles ${ }^{47}$ und Oregon vs. Elstad ${ }^{48}$ wurde die Reichweite des Verwertungsverbots festgelegt. Es sollen demnach Beweise, die nur durch die Aussage des nicht belehrten Beschuldigten gefunden werden, nicht im Prozess verwertet werden. Diese sogenannte "fruits of the poisonous tree doctrine ${ }^{49}$ wurde aber zugleich wieder stark eingeschränkt. Beweise, die auch auf normalem Wege ohnehin entdeckt worden wären, sollen verwertbar sein, damit das Strafverfahren nicht durch den kleinen Belehrungsfehler zum Erliegen kommt. ${ }^{50}$ Diese unbestimmte Auslegung der Fernwirkung von rechtswidrig erlangten Beweismitteln führte dazu, dass im jeweiligen Einzelfall festgestellt werden muss, ob das Beweismittel noch ordnungsgemäß gefunden worden wäre und wie stark der Verstoß gegen die Miranda- oder Beschuldigtenrechte war. Nur so kann dann ein Rückschluss auf die Verwertbarkeit gezogen werden. Der Staatsanwaltschaft obliegt es dann, zu beweisen, dass keine »Frucht des vergifteten Baums « vorliegt. ${ }^{51}$

\section{Auswirkungen der Entscheidungen auf die einzelnen Verfahrensstadien}

Mit der Verhaftung eines Verdächtigen beginnt im amerikanischen Strafprozess das Ermitt- lungsverfahren. ${ }^{52}$ Polizei und Staatsanwaltschaft sind an strenge formelle und materielle Voraussetzungen gebunden, wann eine Festnahme erfolgen darf. ${ }^{53}$ Dies sind zum Beispiel das Vorliegen wahrscheinlicher Tatbegehung, ein Haftbefehl, Waffenanwendung nur bei Gefahr für Leib und Leben des beauftragten Polizeibeamten, oder ein Eindringen in Häuser nur in zwingenden Fällen. ${ }^{54}$ Der Beschuldigte ist bei dieser Festnahme im Rahmen der Miranda- Warnings zu belehren Er muss seine Rechte verstehen und im Stande sein, sie auszuüben. ${ }^{55}$ Erfolgt dann eine selbstbelastende Aussage, ist diese im Strafprozess verwertbar. Es ist darauf zu achten, ob nicht in bestimmten Fällen ein Pflichtverteidiger hinzuzuziehen ist, damit der Beschuldigte effektiv von seinem Verteidigungsrecht Gebrauch machen kann. ${ }^{56}$ Im Anschluss an die Verhaftung ist der Beschuldigte unverzüglich einem magistrate, einem judical officer oder einem coroner vorzuführen. ${ }^{57}$ Dort wird er erneut über seine Rechte belehrt und über den weiteren Gang des Verfahrens und seine diesbezüglichen Entscheidungsmöglichkeiten informiert. Eine weitere Vorprüfung erfolgt in schweren Fällen durch eine grand jury, durch die insbesondere die Haltbarkeit der erhobenen Vorwürfe und die Verwertbarkeit von Geständnissen geprüft wird. Eine Aussage muss der Beschuldigte nicht machen. Im Anschluss an dieses Stadium besteht die Möglichkeit eines Vergleichs zwischen Staatsanwaltschaft und Beschuldigtem, dem sogenannten plea bargaining. ${ }^{58}$ Bei einem guiltyplea $^{59}$ des Beschuldigten (Schuldeingeständnis) kann die Staatsanwaltschaft als Gegenleistung bindende Zugeständnisse hinsichtlich der angeklagten Delikte oder des Strafmaßes machen. ${ }^{60}$ Hier ist die Freiwilligkeit des Schuldbekenntnisses und die Achtung aller Beschuldigtenrechte sicherzustellen. Der Beschuldigte muss von einem Verteidiger vertreten werden. ${ }^{61}$ Auch überprüft der Richter, ob sich der Angeklagte im vollem Bewusstsein der Konsequenzen und ohne Zwang für diesen Vergleich entschieden hat. ${ }^{62}$ Ein Zeichen für Unfreiwilligkeit ist ein unerfüllbares Versprechen seitens der Staatsanwaltschaft oder die Beibringung falscher Beweise vor der Jury. ${ }^{63}$ Der Beschuldigte wird demnach vor jeder von ihm zu treffenden Entscheidung oder Stellungnahme erneut auf seine Rechte hingewiesen oder die Wahrung seiner Rechte wird überprüft. $\mathrm{Zu}$ Beginn der Hauptverhandlung wird dem Angeklagten Gelegenheit gegeben, sich zu den gegen ihn erhobenen Vorwürfen zu äußern oder zu schweigen. Im Falle des Bekennens auf »unschuldig« geht die Verhandlung weiter. Er braucht in diesem Verfahren aber nicht auszusagen. Im Falle des Schuldbekenntnisses erfolgt direkt ein Schuldspruch ohne weitere Verhandlung. Der Richter hat dabei darauf zu achten, dass der Angeklagte die Tragweite seiner Entscheidung versteht und diese freiwillig abgibt. ${ }^{64}$ Auch ist meistens eine anwaltliche Vertretung zwingend vorgeschrieben. ${ }^{65}$ 


\section{Fazit}

Die Rechte des Beschuldigten werden in den Vereinigten Staaten besonders intensiv geschützt. Es wird in allen Verfahrensstadien darauf geachtet, dass der Beschuldigte nicht zu einer Aussage gezwungen wird oder sich durch eine unfreiwillige Aussage selbst belastet. Die Ermittlungsbehörden müssen einen Täter legal überführen, um nicht den gesamten Prozess gegen ihn zu verwirken. Alle geschilderten Versuche der Umgehung dieser strengen Reglementierung wurden zunichte gemacht. Offen geblieben ist aber weiter die Frage, ob der Belehrungstext eine bestimmte strenge Form haben muss und ob es in der Zukunft eine Nachbesserung oder Veränderung des Schutzes geben kann oder wird. Auch bleibt es beim voluntariness test und den zahlreichen genannten Ausnahmen von der Belehrungspflicht. Trotz dieser durchaus weitreichenden Rechte im Rahmen des Beschuldigtenschutzes sollte zur Wahrung der Rechtseinheit und Rechtspraxis in Europa das Amerikanische System nicht übernommen werden. Der zum Vergleich in der BRD existierende und auf die Grundrechte gestützte nemo tenetur Grundsatz ist zugleich eine Ausgestaltungen des politischen Selbstverständnisses und Denkens eines europäischen Staates und in ein rechtsstaatliches Gesamtgefüge eingebaut, das auch einen umfassenden Beschuldigtenschutz ermöglicht. In den USA wird mehr der kontradiktorische Prozess in den Vordergrund gestellt. Sowohl Verteidigung als auch die Staatsanwaltschaft bemühen sich mit allen Mitteln, die Schuld bzw. die Unschuld des Angeklagten zu beweisen, und so den Richter oder die Jury zu überzeugen. Eine herausragende Stellung des Richters, wie zum Beispiel in der $\mathrm{BRD}$, wird dem System nicht zugesprochen. Europa sollte hier einen eigenen Weg anhand seiner eigenen Rechtssysteme und Erfahrungen wählen und nicht die starre und zum Teil unsichere Prozesspraxis der USA übernehmen.

Der Verfasser ist Rechtsanwalt für Straf- und Strafprozessrecht in der Kanzlei Dr. Hüsch\& Partner in Neuss.

\section{Fußnoten:}

1 Schmid, Niklaus: Strafverfahren und Strafrecht in den Vereinigten Staaten, 2. Auflage 1999, S. 125f; Rogall, Klaus: Der Beschuldigte als Beweismittel gegen sich selbst, Dissertation Bonn 1976 S. 81, 82; Hirschberg, Max: Das amerikanische und deutsche Strafverfahren in rechtsvergleichender Sicht, 1. Auflage Neuwied 1963, S. 43.

2 Zur Bedeutung: Verrel, Torsten: Die Selbstbelastungsfreiheit im Strafverfahren, 1. Auflage München 2001, S. 1-3.

3 sogenannte »Selbstbezichtigungsfreiheit «; Makrutzki, Partic: Verdeckte Ermittlungen im Strafprozess, rechtsvergleichende Studie mit dem US- amerikanischen Prozessrecht, 1. Auflage Berlin 2000, S. 297.

4 Auszug aus Amendment V: »... No person.. shall be complelled in any case to be a witness against himself...«.

5 Rogall, Der Beschuldigte (o. Fußn. 1) S. 84.
6 Auch "fundamental fairness « genannt; Hampton vs. US 425 U.S. 484, 494; Makrutzki, S. 297.

7 Wortlaut der Klausel:».. nor shall any State deprive any person of life, liberty, or property without due process of law..

8 Auszug aus Amendment XIV: "... nor deny any person within its jurisdiction the equal protection of the laws."

9 Beispielhaft aus dem Jahr 1943: McNabb vs. United States 318 US 332, 63 S.Ct. 608, 87 L. Ed. 819.

10 Hay, Peter: US- Amerikanisches Recht, 2. Auflage München 2003, RN 604, 605.

11 Hay, aaO., RN. 604; Ransiek, Andreas: Die Rechte des Beschuldigten in der Polizeivernehmung, 1. Auflage Heidelberg 1990, S. 42; Schmid, (o. Fußn. 1) S. 131

12 Trüg, Gerson: Lösungskonvergenzen trotz Systemdivergenzen im deutschen und US- amerikanischen Strafverfahren, 1 . Auflage Tübingen 2003, S. 416, 417; Hopt vs. Utah 110 U.S. 574 4S.Ct. 202, 28 L. Ed. 262 (1884).

13 auch trustworthiness genannt.

14 Brown vs. Mississippi 297 U.S. 278 (1936).

15 Ransiek, Rechte des Beschuldigten (o. Fußn. 8) S. 24.

16 Chambers vs. Florida 309 U.S. 227 (1940); Haley vs. Ohio 332 U.S. 596 (1948): Beschuldigter nur 15 Jahre alt.

17 Ransiek, Rechte des Beschuldigten (o. Fußn. 8) S. 24.

18 Escobedo vs. Illinois 378 U.S. 478 (1964).

19 Auszug aus Amendment VI: "... In all criminal prosecutions, the accused shall enjoy the right... and to have the Assistance of Councel for his defence

20 Schmid, (o. Fußn. 1) S. 125

21 Miranda vs. Arizona, 384 U.S. 436 (1966).

22 Vignera vs. New York: Beschuldigter wurde mehrmals verhört und in verschiedene Polizeireviere verbracht; dann erst formelle Festnahme; Quelle: 384 U.S. 436 (1966).

23 Westover vs. United States: Dauerverhöre durch Polizei und FBI; Quelle: 384 U.S. 436 (1966)

24 California vs. Stewart: Festnahme im eigenen Haus und Hausdurchsuchung ohne Erlaubnis; Festnahme der gesamten Familie und fünftägiges Dauerverhör; nach Geständnis erst Vorführung beim Haftrichter; Quelle: 384 U.S. 436 (1966)

25 Auszug aus Amendment V: "... No person.. shall be complelled in any case to be a witness against himself...".

26 Schmid, (o. Fußn. 1) S. 131; Salditt, Franz: 25 Jahre Miranda, GA 1992, 51- 75, S. 56.

27 Originaltext aus der Entscheidung: Prior to any questioning, the person must be warned that he has a right to remain silent, that any statement he does make may be used as evidence against him, and that he has a right to the presence of an attorney, either retained or appointed.«, aus: Schmid, (o. Fußn. 1) S. 131; Gerson (o. Fußn. 12) S. 419.

28 Ransiek, Rechte des Beschuldigten (o. Fußn. 8) S. $38,39$.

29 Gerson, (o. Fußn. 12) S. 422.

30 Schmid, (o. Fußn. 1) S. 131

31 Hay, (o. Fußn. 7) RN. 613.

32 Hay, aaO. RN. 613, Gerson, (o. Fußn. 12) S 421.

33 Schmid, (o. Fußn. 1) S. 131, insbesondere dort Fußnote 134

34 Dickerson vs. United States 530 U.S. 428 (2000); Gerson (o. Fußn. 12) S. 418 und Teil V Kap. 2, 6. III 8.

35 Gerson, (o. Fußn. 12) S. 441

36 »Miranda, being a constitutional decision of this Court, may not be in effect overruled by an Act of Congress.« Quelle: www.findelaw.com.
37 Gerson (o. Fußn. 12) S. 426, 427.

38 Gerson (o. Fußn. 12) S. 432.

39 Salditt, (o. Fußn. 22) S. 58; Oregon vs. Elstad 84 L. Ed. 2d 222 (1985).

40 Schmid, (o. Fußn. 1) S. 138, Gerson (o. Fußn. 12) S. 427.

41 Ransiek, Rechte des Beschuldigten (o. Fußn. 8) S. 43.

42 Pennsylvania vs. Union Gas Co. 491 U.S. 1, 45 (1989); Salditt (o. Fußn. 22) S. 63.

43 Schmerber vs. California 384 U.S. 757 (1966); Andresen vs. Maryland 427 U.S. 463 (1976); U.S. vs. Wade 388 U.S. 218 (1967); Gilbert vs. California 388 U.S. 263 (1967).

44 Schnabl, Robert: Der O.J. Simpson- Prozess, 1. Auflage Berlin 1999, S. 83; Simpson wurden anstatt der seitens der Verteidigung geforderten 3 Haare sogar durch gerichtlichen Beschluss 100 Haare zur Genanalyse entnommen.

45 Salditt, aaO. S. 59

46 Ausnahme: der sog. Impeachment- Prozess, Harris vs. New York 401 U.S. 222 (1971).

47 New York vs. Quarles 467 U.S. 649 (1984).

48 Oregon vs. Elstad 470 U.S. 298, 105S. Ct. 1285, 84 L. Ed. 2d222 (1985); Gerson (o. Fußn. 11) S. 431..

49 Bosch, Nikolaus: Aspekte des nemo- teneturPrinzips aus verfassungsrechtlicher und strafprozessualer Sicht, 1. Auflage Berlin 1998, S. 347; Schnabl, (o. Fußn. 44) S. 130.

50 Wong Sun vs. United States 371 U.S. 471 (1963); also vor der Miranda- Entscheidung! 51 Salditt, (o. Fußn. 22) S. 62.

52 Hay, (o. Fußn. 7) RN. 607; Hirschberg, (o. Fußn. 1) S. 25; Herrmann, Joachim: Der amerikanische Strafprozess, in: Der Strafprozess im Spiegel ausländischer Verfahrensordnungen, $S$. 133ff, Hrsg. Hans Jung, 1. Auflage Berlin 1990, S. $135,136$.

53 Nach manchen Gerichten müssen immer die Voraussetzungen für einen warrent (Durchsuchungsbefehl) vorliegen für die Zulässigkeit staatlicher Maßnahmen!, Matrutzki (o. Fußn. 3) S. 295; Gershman in: Yale Law Journal 1982, S. $1587,1588$.

54 Richards vs. Wisconsin, 520 U.S. 385 (1997): hinreichender Verdacht erforderlich; Hay, (o. Fußn. 7) RN. 610.

55 Ransiek, Rechte des Beschuldigten (o. Fußn. 7) S. 35.

56 Ransiek, aaO. S. 35

57 Hay, (o. Fußn. 7) RN. 616

58 Dohr, Manuel: Die Berücksichtigung freiwillig geleisteter Wiedergutmachung des Täters bei der Durchführung des Strafverfahrens durch den amerikanischen Staatsanwalt, 1. Auflage Köln 1998, S. 157, 158.

59 Gerson, (o. Fußn. 12) S. 146, das guilty plear wird einem confession (Geständnis) gleichgestellt, der Beschuldigte fällt sein eigenes Schuldurteil: »a plea of guilty is more than a confession wich admits that the accused did various acts, it is itself a conviction; nothing remains but to give a judgement and determine punishent «, Boykin vs. Alabama 395 U.S. 238, 89 S.Ct. 170923 L.Ed. 2d274 (1969).

601999 wurden 94,4\% aller angeklagten Strafverfahren die zur Verurteilung führten mit einem guilty plear vorzeitig beendet, Gerson (o. Fußn. 12) S. 147

61 Hirschberg, (o. Fußn. 1) S. 15.

62 Hay, (o. Fußn. 7) RN. 620.

63 Waley vs. Johnston, 316 U.S. 101 (1942); Hay, aaO. RN. 620.

64 Ransiek, Rechte des Beschuldigten, (o. Fußn. 8) S. 35.

65 Hirschberg, (o. Fußn. 1) S. 15. 